\title{
DESENVOLVIMENTO DE UM SOFTWARE PARA O DIMENSIONAMENTO DE SISTEMAS DE VENTILAÇÃO E RESFRIAMENTO EVAPORATIVO EM INSTALAÇÕES AVÍCOLAS CLIMATIZADAS
}

\author{
Daniel Gustavo de Pauli ${ }^{1}$; Jadir Nogueira da Silva²; Ricardo Brauer Vigoderis ${ }^{3}$; Ilda de \\ Fátima Ferreira Tinoco ${ }^{4}$; Vladimir Oliveira de lorio $^{5}$; Svetlana Fialho Soria Galvarro ${ }^{1}$
}

\begin{abstract}
RESUMO
Este trabalho foi conduzido com o objetivo de desenvolver um software para o dimensionamento completo de sistemas de resfriamento evaporativo, levando em consideração o balanço de energia dentro das instalações, o qual inclui o calor produzido pelas aves, radiação proveniente da cobertura e outros. Buscou-se, também, simular as condições de conforto dentro das instalações, assim como a temperatura, umidade relativa e demais variáveis psicrométricas do ar interno ao longo do comprimento das instalações. O programa foi desenvolvido na linguagem de programação Object Pascal, utilizando Delphi 6.0, para rodar em ambiente Windows. O programa apresentou-se simples e eficaz, resumindo, em poucas janelas, todos os dados necessários à análise do balanço de energia em um galpão avícola, equipado com sistema de resfriamento evaporativo, fornecendo informações suficientes ao dimensionamento desse sistema de resfriamento. Esse programa será extremamente útil para o correto dimensionamento de Sistemas de Resfriamento Evaporativo, pois, fornece para 10 pontos diferentes (de uma extremidade à outra do galpão) as principais características psicrométricas do ar interno, além do Índice de Temperatura e Umidade, contemplando os acréscimos de calor e massa das aves e de calor da construção. A desconsideração dessas variáveis tem levado a erros de projeto, os quais poderão ser evitados por meio dos subsídios, que o software venha oferecer. Além disso, por meio do programa, pode-se calcular o número de exaustores e definir a disposição, a área e o comprimento de placa porosa necessários, além da velocidade do ar na placa.
\end{abstract}

Palavras-chave: avicultura; conforto térmico; informática.

\section{ABSTRACT \\ Development of software to design ventilation and evaporative cooling systems in climatized poultry facilities}

This work aimed at developing software to entirely design an evaporative cooling system, taking into consideration the energy exchange in the building, which among other factors, includes the heat produced by the birds and radiation from the roofing. It also aimed to simulate the fluctuations of temperature, relative humidity, comfort conditions and other psychometric variables of indoor air along length of the installation. The program was developed with use of Paschal Object language, using Delphi 6.0, to work in Windows. The program was found to be efficient, in summarizing all necessary data, in few windows, for the analysis of energy exchange in an avian facility equipped with an evaporative cooling system, thus providing enough information to design a system for Brazilian facilities. Such a tool would be extremely useful for the correct design of evaporative cooling systems, as it also provides the main psychometric characteristics, air temperature and humidity index, contemplating heat addition, bird mass and heat at 10 different points of the installation. Ignoring these factors has led to design errors, which can be avoided by the use of this software. The program also calculates the number of exhaust fans and indicates their placement, the required area and length of pad material, and the air speed close to the pad.

Keywords: broiler chicken; thermal comfort; information technology

\footnotetext{
Recebido para publicação em 25.11.2005

${ }^{1}$ Estudante de Graduação, UFV - DEA - AMBIAGRO, ambiagro@ufv.br.

${ }^{2}$ Prof. Titular, Ph.D, UFV - DEA, jadir@ufv.br

${ }^{3}$ Prof. Adjunto I_DS , UFRPE/UAG, vigoderis@yahoo.com.br

${ }^{4}$ Prof. Adjunta IV, D.S., UFV - DEA - AMBIAGRO, iftinoco@ufv.br

${ }^{5}$ Prof. Adjunto _, D.S., UFV - DCC, vladimir@dpi.ufv.br
} 


\section{INTRODUÇÃO}

Visando suprir quantitativamente o mercado consumidor, tornou-se necessário elevar a produção animal a nível industrial. Para aumentar a produtividade e otimizar espaço e energia, optou-se por um aumento no número de animais por área, ocasionando alterações, que afetam diretamente a qualidade de vida desses animais. Este problema se agrava ainda mais, quando a produção ocorre em locais onde há altas temperaturas ambientais, como é o caso de algumas regiões brasileiras.

As altas temperaturas atuam negativamente na produção animal, impedindo que este expresse seu máximo potencial genético, causando redução na ingestão de ração e, conseqüentemente, no ganho médio de peso e aumento na mortalidade, prejudicando, portanto, a conversão alimentar e os índices de eficiência produtiva esperada (MATOS, 2001; SOUZA, 1996; COOPER e WASHBURN, 1998; CURTIS,1983).

Para sanar os problemas causados pelas altas temperaturas, há vários sistemas de ventilação e resfriamento, sendo que o mais utilizado no Brasil, atualmente, é o sistema de resfriamento evaporativo, cuja utilização proporciona condições ambientais favoráveis ao desenvolvimento dos animais, possibilitandoIhes expressar totalmente seu potencial genético.

Contudo, grande parte dos projetos para dimensionamento de sistemas de ventilação e de resfriamento evaporativo apresenta erros, que ocasionam problemas como taxas insuficientes de ventilação e resfriamento insuficiente do ar, além do desperdício de energia e água proveniente da inadequação dos sistemas.

Com o advento da informática, o computador tem se tornado uma ferramenta fundamental para engenheiros, quando se trata de cálculo e implementação de projetos vários softwares têm sido desenvolvidos para esta finalidade. Entretanto, existe uma demanda em relação a softwares, que possam auxiliar o engenheiro no dimensionamento de sistemas de ventilação e resfriamento evaporativo em instalações avícolas, como também a simulação das condições ambientais no interior das mesmas.

Diante do exposto, este trabalho foi realizado com o objetivo de desenvolver um software para o dimensionamento completo de sistemas de resfriamento evaporativo, levando em consideração o balanço de energia dentro das instalações, o que inclui - calor produzido pelas aves, radiação proveniente da cobertura e outros. Buscouse, também, simular o comportamento das condições de conforto dentro das instalações, assim como a temperatura, umidade relativa e demais variáveis psicrométricas do ar interno ao longo do comprimento das instalações.

\section{MATERIAL E MÉTODOS}

O programa foi desenvolvido na linguagem de programação Object Pascal, utilizando-se Delphi 6.0, para rodar em ambiente Windows.

Em condições de regime permanente, em que não há acúmulo de energia no volume de controle, como no caso de um galpão de frangos, o balanço de energia sensível pode ser escrito como:

$$
\mathrm{E}_{\text {entra }}+\mathrm{E}_{\text {animal }}-\mathrm{E}_{\text {sai }}=0
$$

em que

$E_{\text {entra }}=$ energia sensível que entra no volume de controle, W;

$E_{\text {animal }}=$ energia sensível dissipada produzida pelo animal, W; e

$E_{\text {sai }}=$ energia sensível que sai do volume de controle, W.

ALBRIGHT (1990) propõe que o calor sensível, que entra no ambiente, pode ser expresso da seguinte forma:

$$
\begin{aligned}
& \mathrm{E}_{\text {entra }}=\mathrm{q}_{\mathrm{m}}+\mathrm{q}_{\mathrm{so}}+ \\
& +\mathrm{q}_{\mathrm{h}}+\mathrm{q}_{\mathrm{s}}+\mathrm{q}_{\mathrm{w}}+\mathrm{q}_{\mathrm{f}}+\mathrm{q}_{\mathrm{vi}}
\end{aligned}
$$

em que

$\mathrm{q}_{\mathrm{m}}=$ calor sensível proveniente de fontes mecânicas, elétricas e outras, tais como os motores e luzes, $\mathrm{W}$;

$\mathrm{q}_{\mathrm{so}}=$ calor sensível resultante da absorção da radiação solar no interior da instalação, W;

$\mathrm{q}_{\mathrm{s}}=$ calor sensível liberado pelos animais, $\mathrm{W}$;

$\mathrm{q}_{\mathrm{h}}=$ calor sensível proveniente dos sistemas

de aquecimento, $W$; 
$\mathrm{q}_{\mathrm{w}}=$ calor sensível transferido através da estrutura da construção, como do telhado, das paredes, das cortinas, das portas, do horizonte, etc.

$q_{f}=$ calor sensível transferido para o piso, através da região próxima ao perímetro da construção. Normalmente, este tipo de transferência de calor é considerado desprezível; e

$\mathrm{q}_{\mathrm{vi}}=$ calor sensível contido no ar de ventilação, $W$.

De acordo com CASTELLO (1970), o calor sensível dissipado pelas aves foi determinado pela equação

$$
\mathrm{E}_{\text {animal }}=\mathrm{q}_{\mathrm{s}}=\mathrm{q}_{\mathrm{si}} \cdot \mathrm{N} \cdot \mathrm{A}_{\text {piso }}
$$

em que

$\mathrm{q}_{\mathrm{si}}=$ calor sensível liberado por animal, W.ave ${ }^{-1}$;

$\mathrm{N}=$ densidade de criação, aves. $\mathrm{m}^{-2}$; e

$\mathrm{A}_{\text {piso }}=$ área do piso, $\mathrm{m}^{2}$.

O calor sensível dissipado por frangos de corte, criados soltos sobre cama, foi determinado por STROM E FEENSTRA (1980), empregando a equação

$$
\mathrm{q}_{\mathrm{si}}=3 \cdot\left(\mathrm{P}_{\mathrm{c}}\right)^{0,5}
$$

em que

$\mathrm{Pc}=$ massa corporal das aves, $\mathrm{kg}$.

Segundo ALBRIGHT (1990), o calor sensível dissipado pelas aves é resultado do seu metabolismo, sendo que o calor produzido é produto da mantença, do crescimento e da produção das aves.

De acordo com ALBRIGHT (1990), o calor sensível que sai da instalação pode ser expresso da seguinte forma:

$\mathrm{E}_{\mathrm{sai}}=\mathrm{q}_{\mathrm{e}}+\mathrm{q}_{\mathrm{vo}}$

em que

$\mathrm{q}_{\mathrm{e}}=$ taxa de conversão de calor sensível para calor latente no volume de controle considerado; e

$\mathrm{q}_{\mathrm{vo}}=$ calor sensível contido no ar de ventilação, que deixa o volume de controle considerado, referenciado à mesma temperatura que $\mathrm{q}_{\mathrm{vi}}$.

Assim, o balanço de energia sensível dentro do galpão é expresso pela equação (6), sendo ilustrado na Figura 1.

$\mathrm{q}_{\mathrm{m}}+\mathrm{q}_{\mathrm{so}}+\mathrm{q}_{\mathrm{h}}+\mathrm{q}_{\mathrm{s}}+\mathrm{q}_{\mathrm{w}}+$

$+\mathrm{q}_{\mathrm{f}}+\mathrm{q}_{\mathrm{vi}}-\mathrm{q}_{\mathrm{e}}-\mathrm{q}_{\mathrm{vo}}=0$

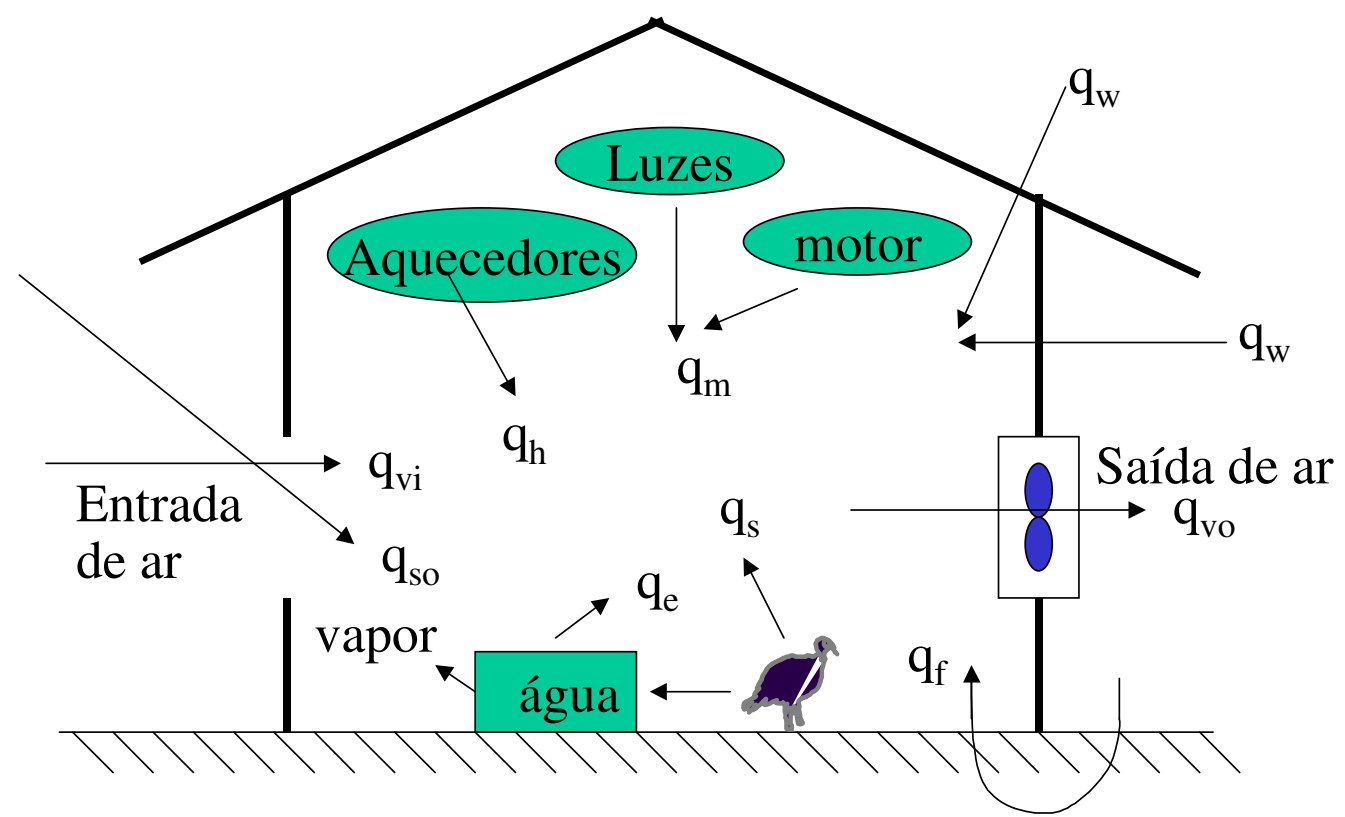

Figura 1. Representação esquemática do balanço de energia sensível dentro de um galpão avícola. 
O calor sensível, transferido pela estrutura da instalação, foi obtido por

$$
\mathrm{q}_{\mathrm{w}}=\sum_{\mathrm{j}=1}^{\mathrm{m}} \mathrm{U}_{\mathrm{i}, \mathrm{j}} \cdot \mathrm{A}_{\mathrm{i}, \mathrm{j}} \cdot\left(\mathrm{t}_{\mathrm{bs}, \mathrm{o}}-\mathrm{t}_{\mathrm{bs}, \mathrm{i}}\right)
$$

em que

$\mathrm{U}_{\mathrm{i}, \mathrm{j}}=$ coeficiente global de transferência de calor, ou condutância, para o i-ésimo volume de controle e j-ésimo componente da estrutura da instalação, $\mathrm{W} / \mathrm{m}^{2} . \mathrm{K}$;

$A_{i j}=$ àrea associada ao i-ésimo volume de controle e j-ésimo componente estrutural da instalação, $\mathrm{m}^{2}$; $t_{\mathrm{bs}, \mathrm{o}}=$ temperatura do ar do ambiente externo, ${ }^{\circ} \mathrm{C}$; e

$t_{b s, i}=$ temperatura do ar no interior do galpão, ${ }^{\circ} \mathrm{C}$.

De acordo com ALBRIGHT (1990), o calor transferido para o piso do edifício ocorre, principalmente, na região do perímetro. Este ganho de calor é proporcional ao perímetro do edifício e à diferença de temperatura entre o ar do interior do galpão e do ambiente externo. Em condições de regime permanente, o ganho de calor pelo piso pode ser determinado por

$$
\mathrm{q}_{\mathrm{f}}=\mathrm{F} \cdot \mathrm{P} \cdot\left(\mathrm{t}_{\mathrm{o}}-\mathrm{t}_{\mathrm{i}}\right)
$$

em que

$\mathrm{F}=$ fator de ganho de calor obtido experimentalmente, $\mathrm{W} \cdot \mathrm{m}^{-1} \cdot \mathrm{K}^{-1}$; e

$\mathrm{P}=$ perímetro do galpão de frangos, $\mathrm{m}$.

Segundo ALBRIGHT (1990) e MEDEIROS (1997), especialmente nos galpões de frangos de corte em que serão utilizadas "camas" isolantes, a quantidade de calor trocada entre o piso e as aves pode ser considerada desprezível em relação às trocas entre os outros elementos construtivos, tais como telhado, paredes e cortinas.
Para determinar o balanço de energia no interior do galpão, este foi dividido em volumes de controle, conforme esquema apresentado na Figura 2.

O galpão foi dividido em até dez volumes de controle, ou seja, as condições do ar foram simuladas para, no máximo, dez pontos da instalação. A temperatura do ar que entra no primeiro volume de controle após atravessar o material poroso $\left(\mathrm{t}_{\mathrm{bs}, 1}\right)$, conforme representado na Figura 1, foi determinada a partir da fórmula de eficiência do resfriamento evaporativo, proposta pela ASHRAE (1996). Desta forma, o comprimento do primeiro volume de controle foi considerado como igual ao comprimento total dos painéis de material poroso, localizados nas laterais da extremidade de entrada do ar.

$t_{b s, 1}=t_{b s, o}-\frac{f}{100} \cdot\left(t_{b s, o}-t_{b u, 0}\right)$

em que

$t_{b s, 1}=$ temperatura do ar que deixa o material poroso umedecido, ${ }^{\circ} \mathrm{C}$;

$f=$ eficiência de resfriamento do sistema $(\%)$ fixada em $80 \%$;

$t_{b s, o}=$ temperatura de bulbo seco do ar externo, ${ }^{\circ} \mathrm{C}$; e

$t_{b u, o}=$ temperatura de bulbo molhado do ar externo, ${ }^{\circ} \mathrm{C}$.

A temperatura de bulbo molhado do ar externo é obtida, utilizando-se um método numérico por iteração.

Com as expressões propostas anteriormente para o balanço de energia, segundo ALBRIGHT (1990), determinou-se o modelo, que expressa a temperatura de bulbo seco no interior do galpão, para os subseqüentes volumes de controle.

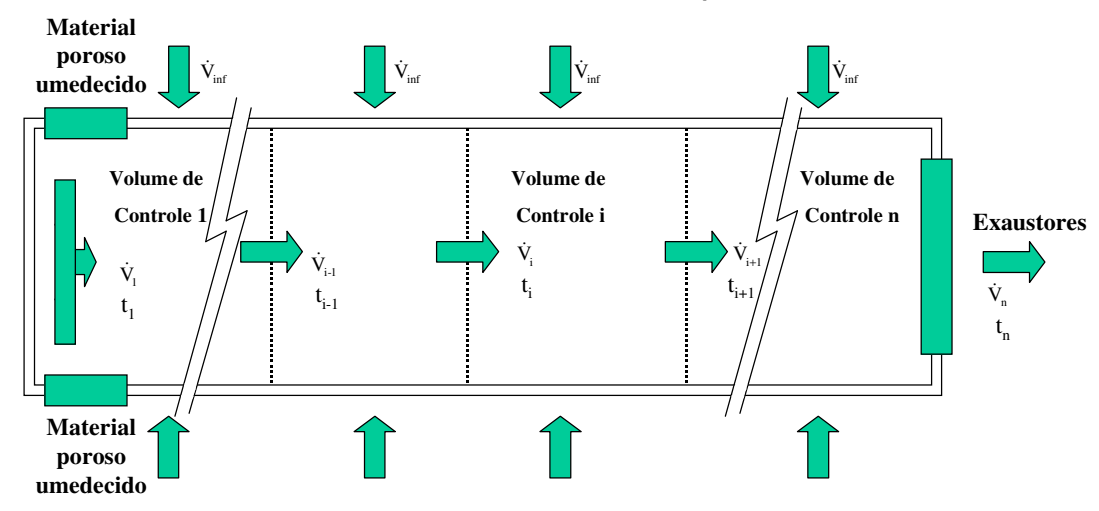

Figura 2. Esquema do balanço de energia sensível em um galpão avícola dividido em $\mathrm{n}$ volumes de controle. (Vista superior) 


$$
\mathrm{t}_{\mathrm{bs}, \mathrm{i}}=\frac{\mathrm{q}_{\mathrm{m}}+\mathrm{q}_{\mathrm{so}}+\mathrm{q}_{\mathrm{h}}+\mathrm{q}_{\mathrm{s}}+\left[\left(\sum \mathrm{UA}+\mathrm{FP}-1006 \cdot \rho_{\text {infi }_{\mathrm{i}}} \cdot \mathrm{V}_{\mathrm{inf}}\right) \cdot \mathrm{t}_{\mathrm{bs}, \mathrm{o}}\right]+1006 \cdot \rho_{\mathrm{i}-1} \cdot \mathrm{V}_{\mathrm{i}-1} \cdot \mathrm{t}_{\mathrm{i}-1}}{\sum_{\mathrm{j}=1}^{\mathrm{m}} \mathrm{U}_{\mathrm{i}, \mathrm{j}} \cdot \mathrm{A}_{\mathrm{i}, \mathrm{j}}+\mathrm{F}_{\mathrm{j}} \cdot \mathrm{P}_{\mathrm{j}}+1006 \cdot\left(\rho_{\mathrm{i}-1} \cdot \mathrm{V}_{\mathrm{i}-1}-\rho_{\mathrm{inf}_{\mathrm{i}}} \cdot \mathrm{V}_{\mathrm{inf}_{\mathrm{i}}}\right)}
$$

em que

$q_{m}=$ calor sensível proveniente de fontes mecânicas, elétricas e outras, como os

motores e luzes, $\mathrm{W}$;

$\mathrm{q}_{\mathrm{so}}=$ calor sensível resultante da absorção da radiação solar no interior da

instalação, W;

$\mathrm{q}_{\mathrm{h}}=$ calor sensível proveniente dos sistemas de aquecimento, $\mathrm{W}$;

$\mathrm{U}_{\mathrm{i}, \mathrm{j}}=$ coeficiente global de transferência de calor, ou condutância, para o i-ésimo

volume de controle e j-ésimo componente da estrutura da instalação, W. $\mathrm{m}^{-2} \cdot \mathrm{K}^{-1}$;

$\mathrm{A}_{\mathrm{i}, \mathrm{j}}=$ área associada ao i-ésimo volume de controle e j-ésimo componente

estrutural da instalação, $\mathrm{m}^{2}$;

$\mathrm{t}_{\mathrm{o}}=$ temperatura do ar do ambiente externo, ${ }^{\circ} \mathrm{C}$;

$\mathrm{t}_{\mathrm{i}}=$ temperatura do ar no interior do galpão, ${ }^{\circ} \mathrm{C}$;

$\mathrm{F}=$ fator de ganho de calor obtido experimentalmente, $\mathrm{W} \cdot \mathrm{m}^{-1} \cdot \mathrm{K}^{-1}$;

$\mathrm{P}=$ perímetro do galpão de frangos, $\mathrm{m}$;

$\rho_{\mathrm{i}-1}=$ densidade do ar baseado nas condições de entrada (i-1); kg.m ${ }^{-3}$;

$\mathrm{V}_{\mathrm{i}-1}=$ vazão volumétrica do ar que entra no volume de controle, $\mathrm{m}^{3} \cdot \mathrm{s}^{-1}$;

$\mathrm{t}_{\mathrm{i}}=$ temperatura no volume de controle $\mathrm{i},{ }^{\circ} \mathrm{C}$;

$\mathrm{t}_{\mathrm{i}-1}=$ temperatura no volume de controle anterior $\mathrm{i}-1,{ }^{\circ} \mathrm{C}$;

$\rho_{\text {inf }}=$ densidade do ar que infiltra no volume de controle; $\mathrm{kg} \cdot \mathrm{m}^{-3}$; e

$\mathrm{V}_{\text {inf }}=$ vazão volumétrica do ar que infiltra no volume de controle, $\mathrm{m}^{3} \cdot \mathrm{s}^{-1}$.

Para o balanço de massa (água + ar), o galpão também foi dividido em até dez volumes de controle, analogamente ao balanço de energia.

No primeiro volume de controle, a umidade foi obtida, logo após a passagem do ar pelo material poroso umedecido, considerando-se a temperatura de bulbo úmido do ar interno igual à do ar externo, segundo ALBRIGHT (1990).

Após passar pelo material poroso umedecido, ou seja, ao deixar o primeiro volume de controle, a umidade inicial do ar de ventilação é alterada devido à umidade produzida pelos animais e à infiltração de ar, que ocorre em cada volume de controle. Desta forma, tem-se

$$
\dot{m}_{i-1}+\dot{m}_{\mathrm{inf}}+\dot{m}_{p}-\dot{m}_{i}=0
$$

em que

$\mathrm{m}_{\mathrm{i}-1}=$ taxa à qual a umidade é carreada para o volume de controle pela ventilação, $\mathrm{kg} . \mathrm{s}^{-1}$;

$\mathrm{m}_{\text {inf }}=$ taxa à qual a umidade é carreada para o volume de controle pela infiltração de ar, kg. $\mathrm{s}^{-1}$;

$\mathrm{m}_{\mathrm{p}}=$ taxa de produção de umidade no interior do volume de controle, $\mathrm{kg} . \mathrm{s}^{-1}$; e

$\mathrm{m}_{\mathrm{i}}=$ taxa à qual a umidade é removida do volume de controle pela ventilação, $\mathrm{kg} \cdot \mathrm{s}^{-1}$.

As vazões mássicas do ar, que entra no volume de controle pela ventilação, pela infiltração e 0 ar que sai do volume de controle podem ser representadas da seguinte forma:

$$
\begin{aligned}
& \dot{m}_{i-1}=\rho_{i-1} \cdot V_{i-1} \cdot W_{i-1} \\
& \dot{m}_{\mathrm{inf}}=\rho_{\mathrm{inf}} \cdot V_{\mathrm{inf}} \cdot W_{\mathrm{inf}} \\
& \dot{m_{i}}=\rho_{i} \cdot V_{i} \cdot W_{i}
\end{aligned}
$$

em que

$\rho_{\mathrm{i}-1}, \rho_{\text {inf }}$ e $\rho_{\mathrm{i}}=$ densidades do ar à temperatura $t_{i-1}, t_{\text {inf }}$ e $t_{i}$, respectivamente, $\mathrm{kg} \cdot \mathrm{m}^{-3}$;

$\mathrm{V}_{\mathrm{i}-1}, \mathrm{~V}_{\mathrm{inf}}$ e $\mathrm{V}_{\mathrm{i}}=$ vazões volumétricas do ar que entra, que infiltra e que sai do volume de controle, respectivamente, $\mathrm{m}^{3} . \mathrm{s}^{-1}$; e

$\mathrm{W}_{\mathrm{i}-1}, \mathrm{~W}_{\text {inf }}$ e $\mathrm{W}_{\mathrm{i}}=$ razões de mistura ou umidades absolutas do ar que entra, que infiltra e que sai do volume de controle, respectivamente, $\mathrm{kg} \cdot \mathrm{kg}^{-1}$.

Assumindo que as densidades do ar, que entra e que sai do galpão, são constantes, obteve-se a razão de mistura para o ar dentro do galpão, a partir da equação 
$\mathrm{W}_{\mathrm{i}}=\frac{\rho_{\mathrm{i}-1} \cdot \mathrm{V}_{\mathrm{i}-1} \cdot \mathrm{W}_{\mathrm{i}-1}+\rho_{\text {inf }} \cdot \mathrm{V}_{\text {inf }} \cdot \mathrm{W}_{\mathrm{inf}}+\mathrm{m}_{\mathrm{p}}}{\rho_{\mathrm{i}-1} \cdot\left(\mathrm{V}_{\mathrm{i}-1}+\mathrm{V}_{\mathrm{inf}}\right)}$

A taxa de produção de umidade no interior do volume de controle $\left(m_{p}\right)$ foi determinada por

$$
\begin{aligned}
& \dot{m}_{p}=m_{p i} \cdot N \cdot A_{p i s o} \\
& \dot{m}_{p i}=2,49 \cdot 10^{-6} \cdot P c^{-0,39}
\end{aligned}
$$

em que

$\mathrm{m}_{\mathrm{pi}}=$ vazão mássica de umidade produzida por ave, $\mathrm{kg} \cdot \mathrm{kg}^{-1} \cdot \mathrm{s}^{-1}$;

$\mathrm{P}_{\mathrm{c}}=$ massa corporal da ave, $\mathrm{kg}$.

$\mathrm{m}_{\mathrm{p}}=$ vazão mássica de umidade produzida, kg.s ${ }^{-1}$;

$\mathrm{N}=$ densidade de criação, aves. $\mathrm{m}^{-2}$; e

$A_{\text {piso }}=$ área do piso, $\mathrm{m}^{2}$.

A umidade relativa do ar, dentro da instalação, foi determinada a partir da razão entre a pressão de vapor $\left(P_{w}\right)$ e a pressão de vapor de saturação $\left(P_{w s}\right)$.

$$
\begin{aligned}
& \mathrm{p}_{\mathrm{w}}=\frac{\mathrm{P}_{\mathrm{atm}} \cdot \mathrm{W}}{0,62198+\mathrm{W}} \\
& \mathrm{p}_{\mathrm{ws}}=0,61078 \cdot 10^{\left(\frac{7,5 \cdot \mathrm{t}_{\mathrm{bs}}}{237,3+\mathrm{t}_{\mathrm{bs}}}\right)}
\end{aligned}
$$

em que

$\mathrm{t}_{\mathrm{bs}}=$ temperatura de bulbo seco, ${ }^{\circ} \mathrm{C}$;

$\mathrm{W}=$ razão de mistura do ar em condições reais, $\mathrm{kg}^{\mathrm{kg}}{ }^{-1}$; e

$\mathrm{P}_{\mathrm{atm}}=$ pressão atmosférica do local, $\mathrm{Pa}$.

Portanto,

$U R=\frac{P w}{P w S} \cdot 100$

A massa específica do ar foi determinada, empregando-se equação proposta por ALBRIGHT (1990), a seguir.

$$
\rho=\frac{1}{\left[(\mathrm{R} \cdot \mathrm{T}) / \mathrm{P}_{\mathrm{atm}}\right] \cdot\left(\frac{1+1,6078 \cdot \mathrm{W}}{1+\mathrm{W}}\right)}
$$

em que

$\rho=$ massa específica, $\mathrm{kg} \cdot \mathrm{m}^{-3}$;

$\mathrm{R}=$ constante dos gases para o ar, $\mathrm{J} \cdot \mathrm{g}^{-1} \cdot \mathrm{K}^{-1}$;

$\mathrm{T}=$ temperatura do ar, $\mathrm{K} ; \mathrm{e}$

$\mathrm{P}_{\mathrm{atm}}=$ pressão atmosférica do local, $\mathrm{Pa}$.

A razão de mistura do ar em condições de saturação $\left(\mathrm{W}_{\mathrm{s}}, \mathrm{kg} \cdot \mathrm{kg}^{-1}\right)$, é dada por

$$
\mathrm{W}_{\mathrm{s}}=0,62198 \cdot \frac{\mathrm{p}_{\mathrm{ws}}}{\mathrm{P}_{\mathrm{atm}}-\mathrm{p}_{\mathrm{ws}}}
$$

A entalpia e o grau de saturação do ar interno para cada volume de controle foram calculados, adicionalmente e determinados a partir das equações a seguir.

$$
\begin{aligned}
& \mathrm{h}=1,006 \cdot \mathrm{t}_{\mathrm{bs}}+\mathrm{W} \cdot\left(2501+1,775 \cdot \mathrm{t}_{\mathrm{bs}}\right) \\
& \mu=\frac{\mathrm{W}}{\mathrm{W}_{\mathrm{s}}}
\end{aligned}
$$

em que

$\mathrm{h}=$ entalpia; $\mathrm{e}$

$\mu=$ grau de saturação, \%.

As condições de conforto para o animal foram analisadas em cada volume de controle do galpão, utilizando-se o índice de conforto proposto por THOM (1959).

A temperatura do ponto de orvalho, necessária para obtenção do valor de ITU, foi determinada por

$$
T_{p o}=\frac{186,4905-237,3 \cdot \log P_{w}}{\log P_{w}-8,2859}
$$

em que

$\mathrm{P}_{\mathrm{w}}=$ pressão de vapor do $\mathrm{ar}$, $\mathrm{mbar}=10^{-2} \mathrm{~Pa}$.

Para realizar a simulação do ar dentro da instalação, algumas hipóteses foram consideradas, conforme apresentado a seguir.

- Regime permanente;

- Os materiais de construção usados são homogêneos, com propriedades constantes;

- A geração interna de calor, no interior do galpão, pelas aves com o mesmo peso é constante;

- Não há calor sensível proveniente de fontes mecânicas, elétricas e outras, tais como os motores e lâmpadas $\left(q_{m}=0\right)$;

- Não há calor sensível resultante da absorção da radiação solar no interior da instalação $\left(\mathrm{q}_{\mathrm{so}}=0\right)$; 
- Não há calor sensível proveniente dos sistemas de aquecimento $\left(q_{h}=0\right)$;

- O calor sensível transferido para o piso, através da região próxima ao perímetro da construção, é desprezível $\left(q_{f}=0\right)$;

- A produção de umidade pelos animais de mesmo peso é constante;

- O fluxo de calor é uniforme;

- A vazão de ar fornecida pelos exaustores é constante;

- A eficiência de resfriamento evaporativo é uniforme, considerada $80 \%$, a qual segundo indicação do fabricante do material poroso, é a mais economicamente viável;

- O coeficiente de convecção é uniforme, nas superfícies externa e interna do galpão;

- A vazão de infiltração de ar no volume de controle foi considerada como sendo $5 \%$ da vazão dentro do galpão, ou vazão de trabalho;

- A espessura do painel poroso foi fixada em $0,15 \mathrm{~m}$, a fim de satisfazer as condições ideais para o funcionamento adequado do sistema, de forma mais viável economicamente.

Considerando 0 regime permanente dentro do galpão, o dimensionamento do sistema ocorre a partir da lei da continuidade, isto é, a vazão de ar é constante ao entrar e sair do galpão.

A área total de painel evaporativa para o galpão foi determinada por

$$
\mathrm{A}_{\text {total }}=\frac{\mathrm{A}_{\text {transversal }} \cdot \mathrm{Vel}_{\text {galpão }}}{\mathrm{Vel}_{\text {placa }}}
$$

em que

$\mathrm{A}_{\text {tota }}=$ área total de placa evaporativa, $\mathrm{m}^{2}$;

$\mathrm{A}_{\text {tranversal }}=$ área da seção transversal do galpão, $\mathrm{m}^{2}$;

$\operatorname{Vel}_{\text {galpão }}=$ velocidade do ar dentro do galpão, m. s $^{-1} ; \mathrm{e}$

$\mathrm{Vel}_{\text {placa }}=$ velocidade do ar ao entrar na placa evaporativa, $\mathrm{m} \cdot \mathrm{s}^{-1}$.

A área da seção transversal do galpão foi obtida por

$$
\mathrm{A}_{\text {tranversal }}=\left[\frac{l^{2} * \operatorname{tg} \alpha}{4}+(l * h)\right]
$$

em que

I = largura do galpão, m;

$\mathrm{h}$ = altura do pé direito do galpão, m; e

$\alpha=$ inclinação do telhado, graus.

A área de placa em cada lateral da extremidade de entrada do ar no galpão foi determinada, dividindo-se a área total pela metade.

Para o dimensionamento do número de exaustores, empregou-se a equação

$$
\mathrm{N}_{\text {exaustores }}^{\text {ox }}=\frac{Q_{\text {trabalho }}}{Q_{\text {exaustor }}}
$$

em que

№ ${ }_{\text {exaustores }}=$ número total de exaustores no galpão; $Q_{\text {exaustor }}=$ vazão de cada exaustor, $\mathrm{m}^{3} \cdot \mathrm{s}^{-1}$; e $Q_{\text {trabalho }}=$ vazão no interior do galpão, $\mathrm{m}^{3} \cdot \mathrm{s}^{-1}$.

Para o dimensionamento do sistema de resfriamento evaporativo, foram feitas as seguintes considerações a seguir:

- Fixou-se a vazão de ar no interior do galpão, ou vazão de trabalho, como sendo a vazão necessária para atingir renovação do ar dentro da instalação, por minuto, pois, como se sabe, esta satisfaz as condições térmicas e higiênicas necessárias ao conforto dos animais;

- A velocidade do ar dentro do galpão foi determinada, em função da vazão de trabalho; e

- A velocidade do ar ao entrar na placa evaporativa, foi obtida a partir dos ábacos do fabricante para uma eficiência do resfriamento evaporativo de $80 \%$.

\section{RESULTADOS E DISCUSSÃO}

Desenvolveu-se um software para o dimensionamento completo de sistemas de resfriamento evaporativo, e também o balanço de energia dentro de instalações avícolas. Visando-se também, simular as variações das condições de conforto dentro das instalações, assim como da temperatura, da umidade relativa, das condições de conforto e demais variáveis psicrométricas do ar interno ao longo das instalações.

$\mathrm{Na}$ figura 3, é apresentado um fluxograma resumido do programa, o qual dá uma idéia global às operações executadas pelo mesmo. 


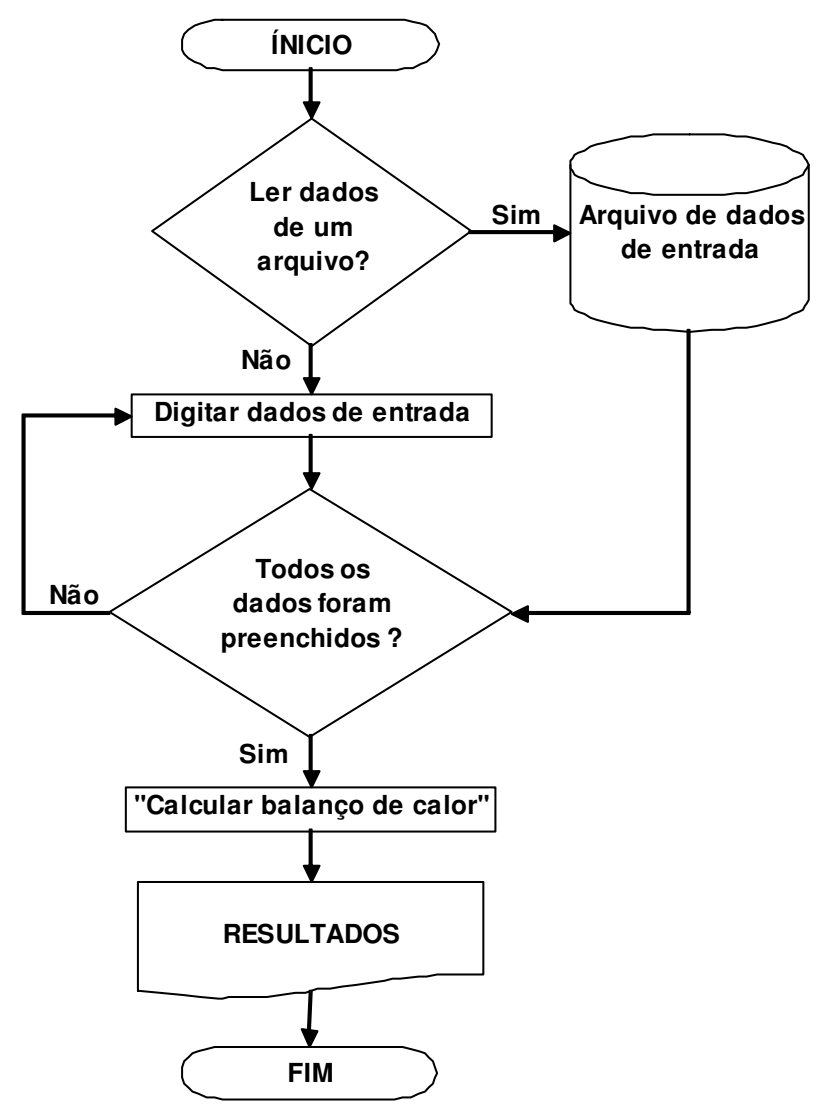

Figura 3. Fluxograma resumido das operações executadas pelo programa.

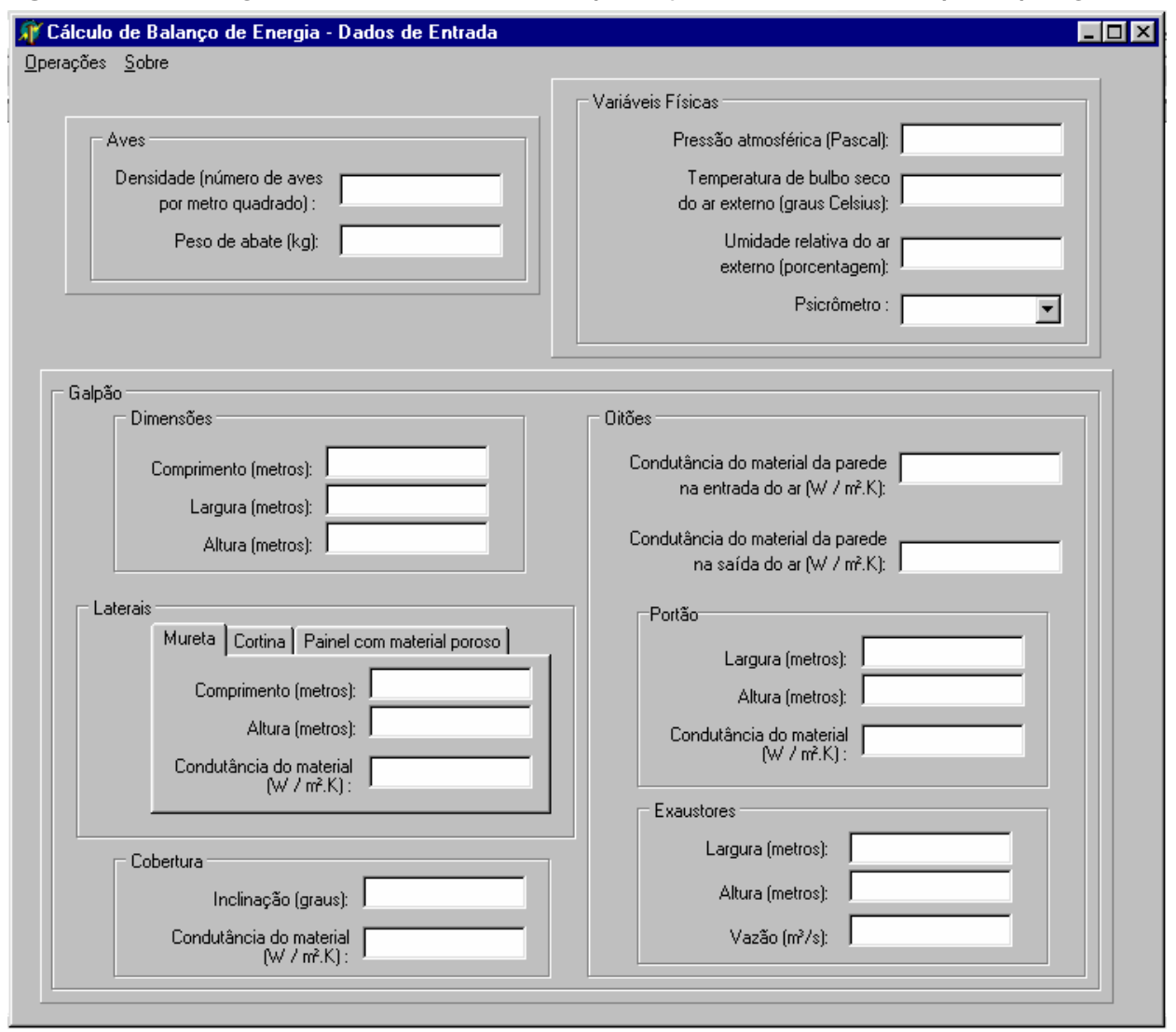

Figura 4. Janela de entrada de dados. 
Na Figura 4, é representada a tela de entrada de dados, onde obrigatoriamente devem-se preencher todos os campos para o cálculo subseqüente.

Os dados de entrada, requeridos pelo programa para os cálculos posteriores, referem-se a informações zootécnicas das aves que serão alojadas, condições psicrométricas do ar de entrada e características do galpão (detalhes construtivos, dimensões e propriedades térmicas de seus materiais).

É disponibilizada, para o usuário, a possibilidade de salvar os valores digitados num arquivo, selecionando-se o item "Salvar dados em um arquivo" do menu "Operações". Por meio do item "Ler os dados de um arquivo", os dados anteriormente salvos podem ser recuperados.

Após o preenchimento dos campos, procede-se ao cálculo, selecionando o item "Calcular balanço de calor" do menu Operações, conforme indicado na Figura 5.

Ao selecionar o item "Calcular balanço de calor", o programa apresentará uma janela de resultados (Figura 6), contendo os dados referentes ao dimensionamento do sistema de resfriamento, bem como uma simulação das condições do ar interno ao longo da instalação, além dos valores de ITU.

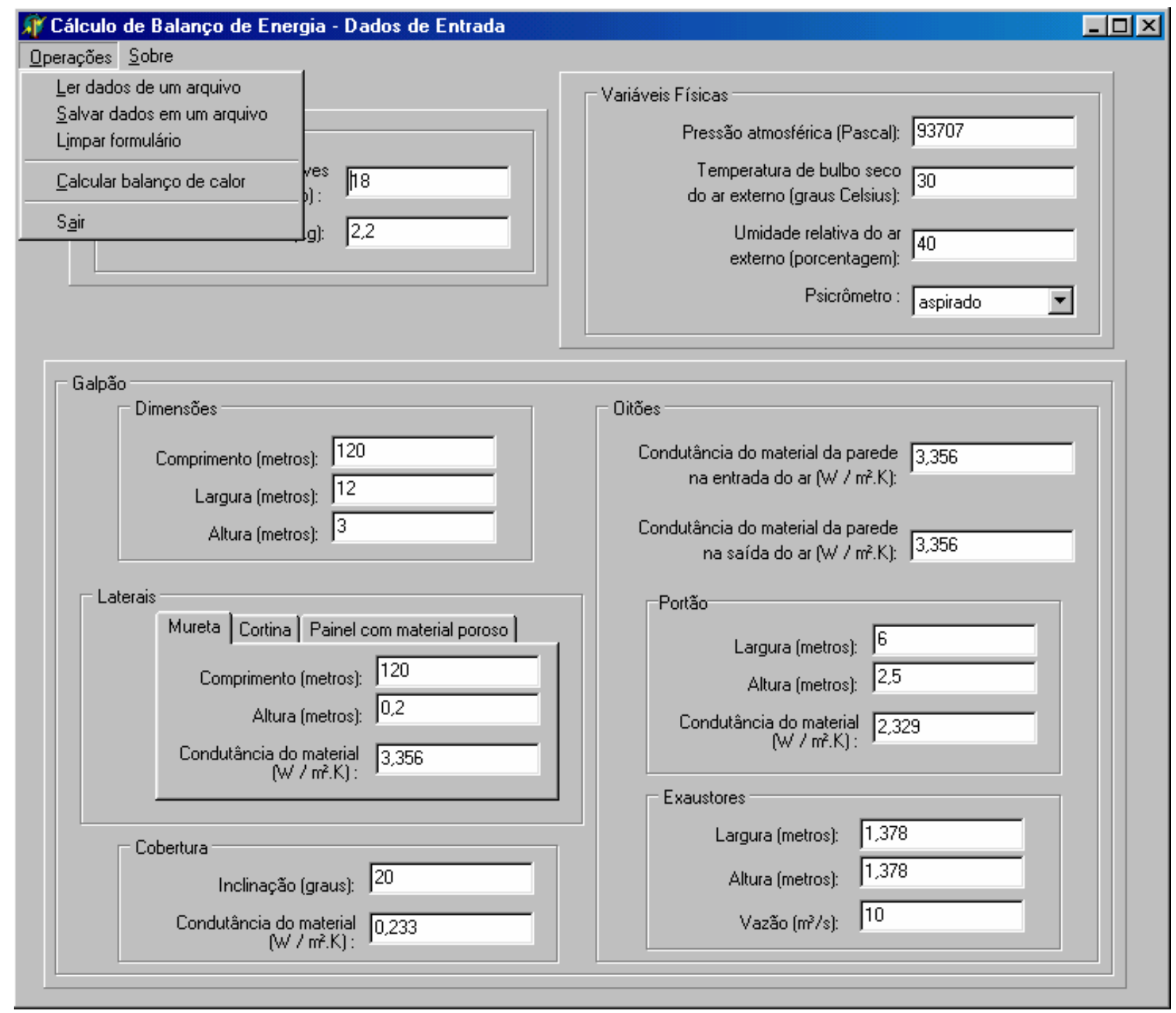

Figura 5. Menu de Operações, mostrando o item "Calcular balanço de calor". 


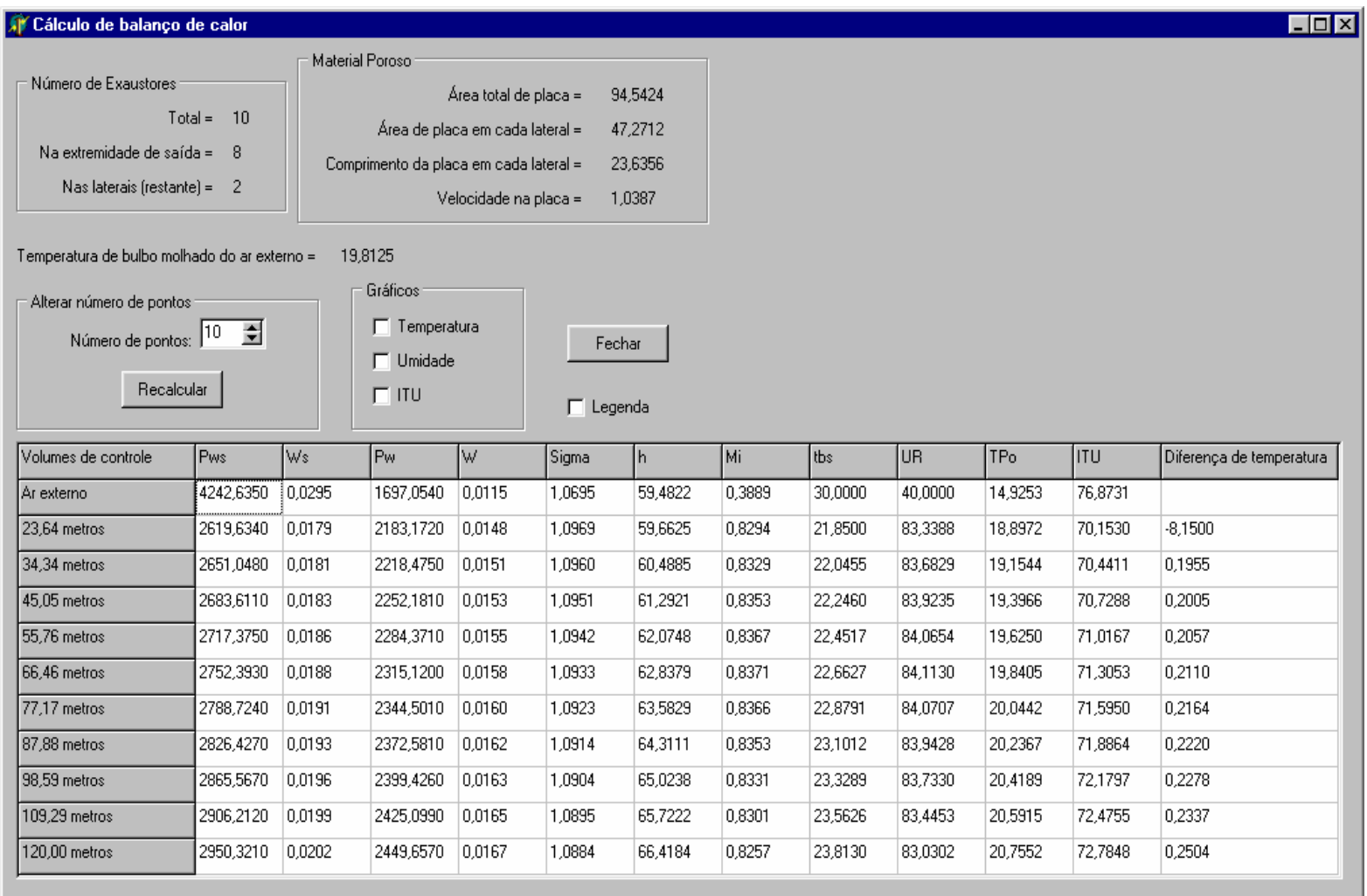

Figura 6. Janela de resultados.

$\begin{array}{ll}\text { 919 Legenda } & \\ \text { PWS } & \text { Pressão de vapor de saturação } \\ \text { WS } & \text { Raxão de mistura de saturação } \\ \text { PW } & \text { Pressão de vapor } \\ \text { W } & \text { Raxão de mistura } \\ \text { Sigma } & \text { Massa especifica } \\ \text { h } & \text { Entalpia } \\ \text { Mi } & \text { Grau de saturação } \\ \text { tbs } & \text { Temperatura de bulbo seco } \\ \text { UR } & \text { Umidade relativa do ar } \\ \text { TPo } & \text { Temperatura do ponto de orvalho } \\ \text { ITU } & \text { Índice de temperatura e umidade }\end{array}$

Figura 7. Legenda contendo a descrição das variáveis apresentadas no balanço de calor. 
Quanto ao dimensionamento do sistema de resfriamento evaporativo, o programa apresenta o número total de exaustores necessários e os distribui na extremidade de saída do ar do galpão. No que diz respeito aos painéis de material poroso, é apresentada a área total do painel necessária para o funcionamento adequado do sistema, sendo esse total é distribuído nas laterais da extremidade de entrada do ar, apresentando também o comprimento dos painéis nas laterais e a velocidade do ar ao entrar no painel poroso umedecido. A vazão de ar de cada exaustor e a altura do painel poroso é determinada pelo usuário, na janela de entrada, sendo que para a altura do painel poroso apresenta-se uma lista das alturas comerciais, oferecidas pelo fabricante.

Em relação ao balanço de calor, apresenta-se uma tabela contendo as variáveis necessárias à descrição das condições do ar dentro e fora da instalação. Estas estão dispostas na primeira linha superior da tabela (Figura 6) e são descritas numa legenda, acionada através do item "Legenda" (Figura 7). Na coluna localizada à esquerda da mesma tabela, são apresentadas as distâncias em relação à extremidade de entrada do ar, dos pontos considerados na simulação das condições do ambiente, sendo que essas distâncias são automaticamente determinadas em função do número de pontos.

O número de pontos, considerados no cálculo, pode ser alterado no item "Alterar número de pontos" na janela de resultados (Figura 6), sendo dez o máximo e dois o número mínimo aceitáveis pelo programa.

Marcando-se as opções do item "Gráficos" da janela de resultados (figura 6), são mostradas representações gráficas do comportamento da temperatura de bulbo seco, da umidade relativa, do índice de temperatura e umidade - ITU ao longo da instalação, e do ar externo à mesma (0,00 metros), os quais estão demonstrados nas Figuras 8, 9, e 10, respectivamente.

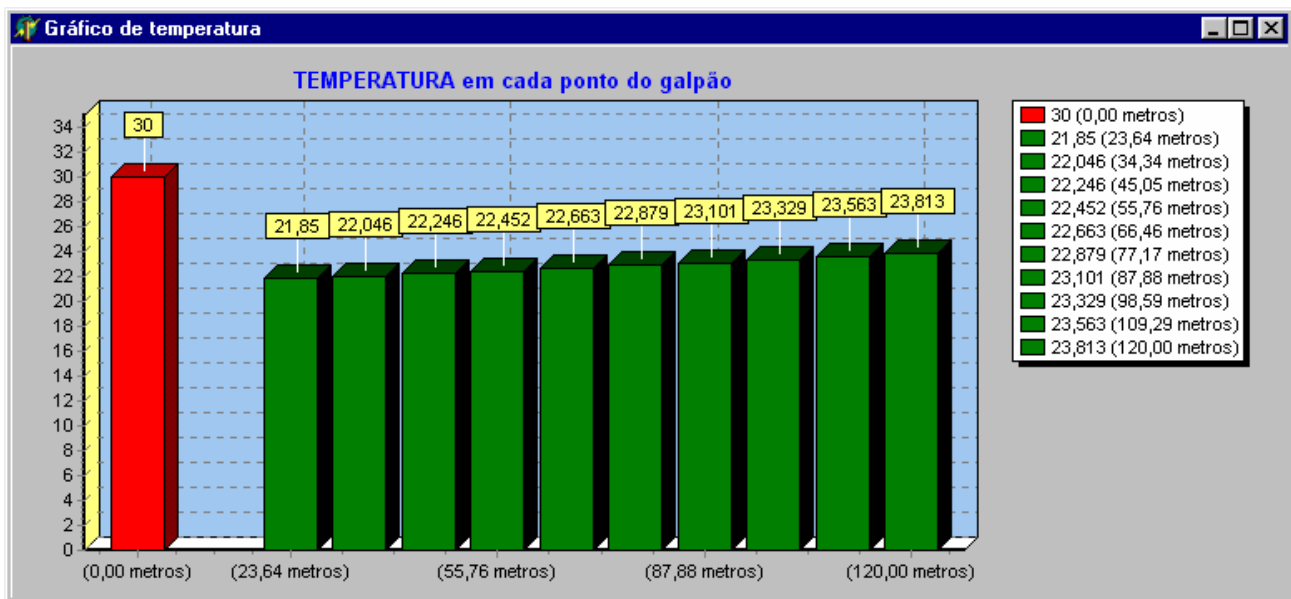

Figura 8. Representação gráfica da variação na temperatura de bulbo seco, ao longo do comprimento do galpão.

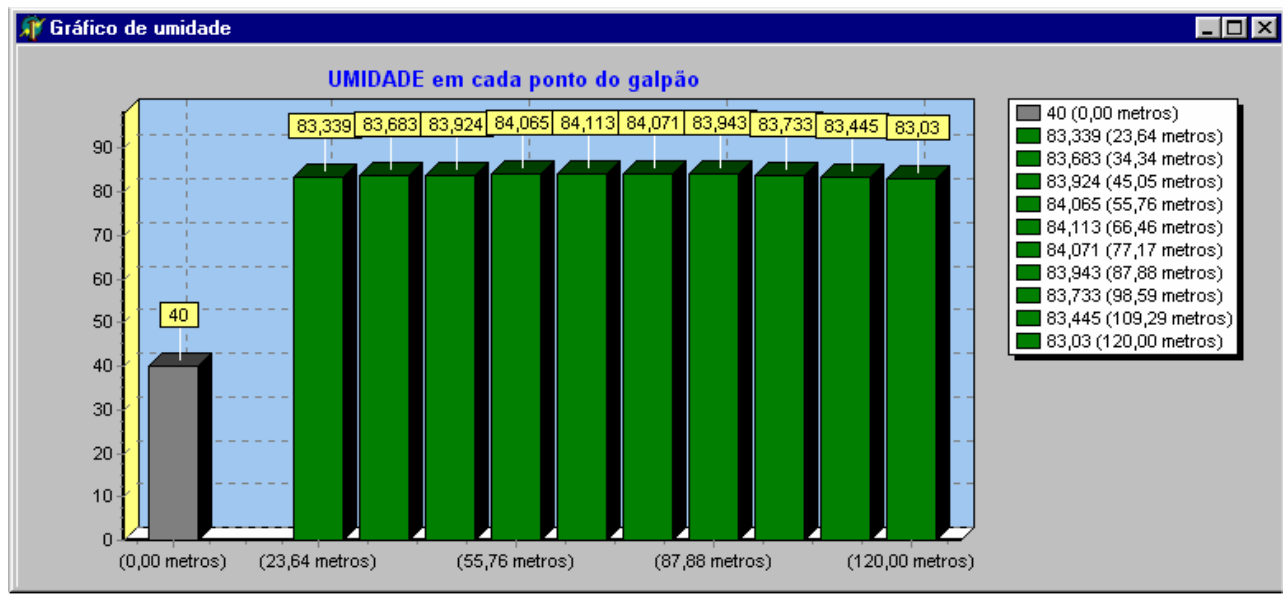

Figura 9. Representação gráfica da variação na temperatura de bulbo seco, ao longo do comprimento do galpão. 


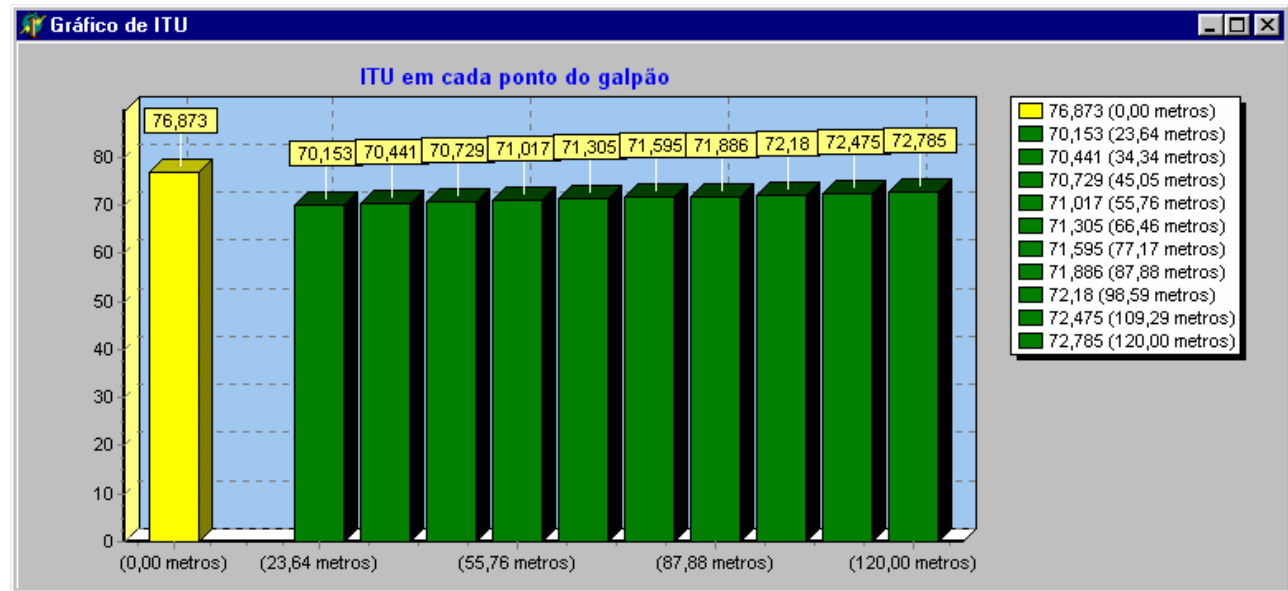

Figura 10. Representação gráfica da variação na temperatura de bulbo seco, ao longo do comprimento do galpão.

Nas figuras 8,9 e 10, as barras de cor verde indicam valores dentro da faixa de conforto para as aves, enquanto, as vermelhas indicam valores acima e as de cor cinza, valores abaixo. Para o ITU (Figura 10) existem barras de cor amarela, as quais indicam uma situação de alerta.

\section{CONCLUSÕES}

Nas condições em que este trabalho foi realizado e conforme os resultados obtidos, conclui-se que o programa é simples e eficiente, resumindo, em poucas janelas, todos os dados necessários à análise do balanço de energia em um galpão avícola, equipado com sistema de resfriamento evaporativo, fornecendo informações suficientes ao dimensionamento do sistema de resfriamento.

Tal ferramenta será extremamente útil para o correto dimensionamento de Sistemas de Resfriamento Evaporativo, pois, fornece para 10 pontos diferentes (de uma extremidade à outra do galpão) as principais características psicrométricas e Índice de Temperatura e Umidade, contemplando os acréscimos de calor e massa das aves, além do calor da construção. Quando não são considerados, estes aspectos podem levar a erros de projeto, os quais poderão ser evitados por meio dos subsídios que o software venha oferecer. Além disso, por meio do programa, pode-se calcular o número de exaustores e definir a disposição, a área e o comprimento de placa porosa necessários e a velocidade do ar na mesma.

\section{REFERÊNCIAS BIBLIOGRÁFICAS}

ALBRIGHT, L.D. Environment control for animals and plants. St. Joseph: ASAE, 1990. $453 p$

\section{ASHRAE System and Equipament Handbook (SI) ASHRAE, 1996.}

CASTELLÓ, J.A. Alojamientos y manejo de las aves. Barcelona: Real Escuela Oficial y Superior de Avicultura, 1970, 79p.

COOPER, M.A.; WASHBURN, K.W. The relationship of body temperature to weight gain, feed consumption, and feed utilization in broilers under heat stress Poultry Science, West Lafauette v.77 p. 237-242, 1998.

CURTIS, S. E. Environmental management in animal agriculture. Ames: The lowa State University Press, 1983. 409p.

MATOS, M.L.; TINÔCO, I.F.F.; BAÊTA, F.C.; CECON, P.R. Conforto térmico ambiente e desempenho de frangos de corte, alojados em dois níveis de alta densidade, em galpões com sistema de ventilação em túnel e ventilação lateral. Viçosa, M.G., UFV, 2001. 89 p. (Dissertação de M.S.). 
MAY, J.D.; LOTT, B.D.; SIMMONS,J.D. The effect of environmental temperature and body weight on growth rate and feed gain of male broilers. Poultry Science, v. 77 p499501, 1998.

MEDEIROS, C.M. Desenvolvimento e aplicação de modelo para simulação e desempenho de galinhas poedeiras e frangos de corte com uso de resfriamento evaporativo. Viçosa: UFV, 1997, 88p. Tese (Mestrado em Engenharia Agrícola - Construções Rurais e Ambiência) - Universidade Federal de Viçosa, 1997.
SOUZA, J.C. Conforto ambiental para aves - ponto de vista do produtor (frangos de corte) In: II SIMPOSIO GOIANO DE AVICULTURA, 1996, Goiânia-GO Anais... , 1996, p.61-62.

STROM, J.S., FEENSTRA, A. Heat loss from cattle, swine and poultry. St. Joseph: American Society of Agricultural Enginneers, 1980, p.75. (Paper 80-4021)

THOM, E.C. The discomfort index. Weatherwise, Boston, v.12, n.1, p.57-60, 1959. 